\title{
Template Instrumentation for "Accurate Constant via Transient Incomplete Separation" (ACTIS)
}

\author{
Jean-Luc Rukundo, ${ }^{1}$ Sven Kochmann, ${ }^{1}$ Tong Ye Wang, ${ }^{1}$ Nikita A. Ivanov, ${ }^{1}$ J. C. Yves Le Blanc, ${ }^{2}$ Boris \\ I. Gorin, ${ }^{3}$ and Sergey N. Krylov ${ }^{1 *}$
}

${ }^{1}$ Department of Chemistry and Centre for Research on Biomolecular Interactions, York University, Toronto, Ontario M3J 1P3, Canada; ${ }^{2}$ SCIEX, Concord, Ontario L4K 4V8, Canada;

${ }^{3}$ Eurofins CDMO Alphora, Mississauga, Ontario L5K 1B3, Canada.

*Corresponding author's email: skrylov@yorku.ca

ABSTRACT: ACTIS is a new method for finding the equilibrium dissociation constant $K_{\mathrm{d}}$ of a protein-small molecule complex based on transient incomplete separation of the complex from the unbound small molecule in a capillary. This separation is caused by differential transverse diffusion of the complex and the small molecule in a pressure-driven flow. The advection-diffusion processes underlying ACTIS can be described by a system of partial differential equations allowing for a virtual ACTIS instrument to be built and ACTIS to be studied in silico. The previous in-silico studies show that large variations in the fluidic system geometry do not affect the accuracy of $K_{\mathrm{d}}$ determination, thus, proving that ACTIS is conceptually accurate. The conceptual accuracy does not preclude, however, instrumental inaccuracy caused by run-to-run signal drifts. Here we report on assembling a physical ACTIS instrument with a fluidic system that mimics the virtual one and proving the absence of signal drifts. Furthermore, we confirmed method ruggedness by assembling a second ACTIS instrument and comparing the results of experiments performed with both instruments in parallel. Despite some differences between the instruments and, accordingly, significant differences in their respective separagrams, we found that the $K_{\mathrm{d}}$ values determined for identical samples with these instruments were equal. Conclusively, the fluidic system presented here can serve as a template for reliable ACTIS instrumentation.

Proteins (P) can bind small-molecule ligands (L) to form stable non-covalent complexes (PL):

$$
\mathrm{P}+\mathrm{L} \underset{k_{\text {off }}}{\stackrel{k_{\text {on }}}{=}} \rightarrow \mathrm{PL}
$$

The stability of PL is characterized by the equilibrium dissociation constant $\left(K_{\mathrm{d}}\right)$ defined as:

$$
K_{\mathrm{d}}=[\mathrm{L}]_{\mathrm{eq}}[\mathrm{P}]_{\mathrm{eq}} /[\mathrm{PL}]_{\mathrm{eq}}
$$

where, $[\mathrm{P}]_{\mathrm{eq}},[\mathrm{L}]_{\mathrm{eq}}$, and $[\mathrm{PL}]_{\mathrm{eq}}$ are equilibrium concentrations of $\mathrm{P}$, $\mathrm{L}$, and PL, respectively. Determining accurate $K_{\mathrm{d}}$ values for PL is important for biology and technology. ${ }^{1}$ All established methods, currently used for finding $K_{\mathrm{d}}$ of PL, have inherent sources of inaccuracy. ${ }^{2}$ Accordingly, $K_{\mathrm{d}}$ values determined by different methods for the same PL complex may differ by orders of magnitude. ${ }^{3}$ Even $K_{\mathrm{d}}$ determined by the same method for the same binding pair can differ drastically. ${ }^{4}$ In the absence of a reference method, $K_{\mathrm{d}}$ values differing by more than 100 times may be considered consistent and kept in one data set. ${ }^{5}$ These large inaccuracies in $K_{\mathrm{d}}$ values inevitably lead to misinterpretation of experimental results, mistaken conclusions, and misconceptions. ${ }^{6}$

We recently introduced Accurate Constant via Transient Incomplete Separation (ACTIS), a method for finding $K_{\mathrm{d}}$ of PL, which was hypothesized to be free of inherent sources of inaccuracy. ${ }^{7}$ ACTIS is based on a long-known phenomenon of transient incomplete separation (TIS) of the L from PL in a roundcross-section capillary due to their differential transverse diffusion in a laminar flow with a parabolic velocity profile. ${ }^{8,9}$ In ACTIS, a short plug of an equilibrium mixture of $\mathrm{P}$ and $\mathrm{L}$ in a buffer solution is injected into a capillary prefilled with the pure buffer solution. The plug is then propagated inside the capillary by a pressure-driven flow of the buffer solution. Different rates of transverse diffusion of PL and $\mathrm{L}$ in the laminar flow cause their TIS in the longitudinal direction (Figure 1A) resulting in a separagram containing two unresolved peaks: a non-diffusive peak for PL and a diffusive peak for L. ${ }^{8,9}$ To determine $K_{\mathrm{d}}$, TIS is performed for a series of equilibrium mixtures (EMs) with a constant concentration of $\mathrm{L}$ and varying concentration of $\mathrm{P}$, producing a set of separagrams (Figure 1B). The cumulative signal of protein-bound and protein-unbound $\mathrm{L}$ is used to build a classic binding isotherm "fraction of free $\mathrm{L}$ versus the concentration of P" which reveals the value of $K_{\mathrm{d}}$ (Figure 1C). ${ }^{2}$

ACTIS is a deterministic method, i.e., the advection-diffusion processes that ACTIS is based upon can be described by a system of partial differential equations with fully defined initial and boundary conditions. The deterministic nature of ACTIS allowed us to create a virtual ACTIS instrument in silico and study the accuracy of ACTIS by computer modeling. ${ }^{7}$ This study proved that the accuracy of ACTIS was not affected by large variations in the geometry of the fluidic path and/or the parameters characterizing the flow. The in-silico study led to several important conclusions. The initial understanding was that a physical ACTIS instrument had to support ideal conditions such as $(i)$ a cylindrical shape of the initial sample plug, (ii) a parabolic flow-velocity profile, and (iii) the absence of ramp time in the pressure pulse. ${ }^{7}$ Maintaining such ideal conditions would require a complex ACTIS instrument and would not leave flexibility with fluidic path design. The invariance of accuracy demonstrated in silico suggests that an ACTIS instrument can be much simpler than it was thought initially. ${ }^{2}$ Furthermore, it suggests that the design of the fluidic system can be changed (if needed) without concerns that the accuracy could be affected. Finally, the conceptual accuracy of ACTIS allows the developers to focus on potential instrumental sources of inaccuracy such as signal drifts caused by run-to-run sample carryover and/or long-term operation instability of instrument components.

Here we report on (i) constructing a physical ACTIS instrument that mimics the previously reported simple virtual instrument, ${ }^{7}$ and (ii) proving the stability of its operation. The fluidic system of this instrument was coupled with a fluorescence detector. Bovine serum albumin (BSA) and fluorescein were the protein and small molecule for binding of which the $K_{\mathrm{d}}$ value was measured. We found no significant signal drifts in this ACTIS instrument. We then assembled the second ACTIS instrument and run experiments in parallel on the two instruments on different days by different operators to prove the instrumental ruggedness of the method. The $K_{\mathrm{d}}$ values obtained for the same samples with the two instruments deviated by no more than $10 \%$. Our results suggest that the simple fluidic system presented here can serve as 


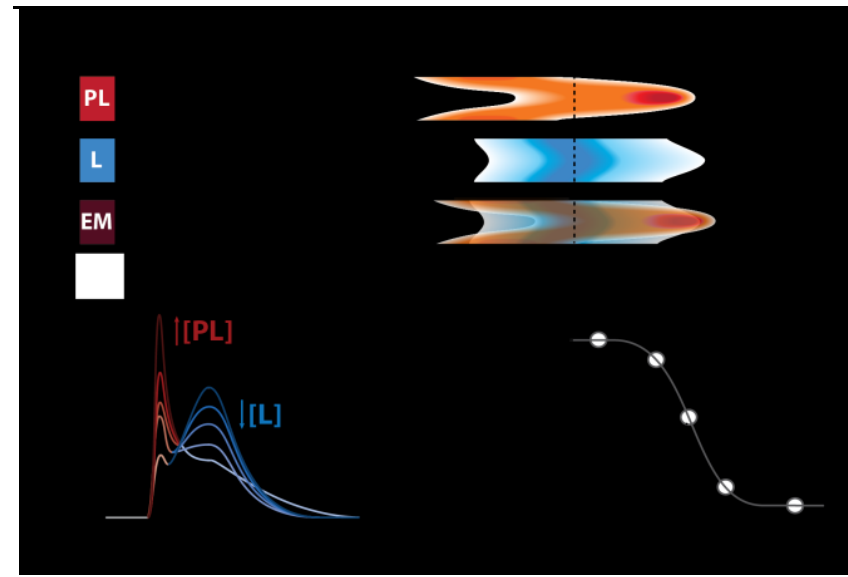

Figure 1. Simplified schematic of determining $K_{\mathrm{d}}$ by ACTIS. A: A short plug of the equilibrium mixture (EM) of $\mathrm{P}$ and $\mathrm{L}$, containing free $\mathrm{P}$, free $\mathrm{L}$, and the complex PL, is pressure-propagated through a capillary. Differences in rates of transverse diffusion of PL and L cause their longitudinal separation. B: Longitudinal separation results in two unresolved peaks: a non-diffusive peak associated with PL and a diffusive peak associated with L. A cumulative signal from L and PL is measured at time $\tau_{\mathrm{L}}$, corresponding to the maximum of the diffusive peak; this is the characteristic time of transverse diffusion of $\mathrm{L}$. The signal is measured at a constant concentration of $\mathrm{L}$ and varied concentrations of P. C: A binding isotherm "normalized signal at time $\tau_{\mathrm{L}} v s$ the total concentration of P" is built, from which the value of $K_{\mathrm{d}}$ is obtained. $R$ is the fraction of free ligand obtained from the normalised signal. This figure is adapted from ref. 7.

a reliable template for ACTIS instrumentation allowing for ACTIS research on practical applications of this new method for $K_{\mathrm{d}}$ determination in protein-small molecule binding studies.

\section{MATERIALS AND METHODS}

Reagents and solutions. All reagents were obtained from Sigma-Aldrich (Oakville, Ontario, Canada). The binding pair of a protein and small-molecule ligand used here was bovine serum albumin (BSA, catalog A2514) and fluorescein sodium salt (catalog F2456). New BSA solutions of 10 concentrations were prepared prior to each ACTIS experiment and used to prepare new EMs. The same stock solution of $1 \mu \mathrm{M}$ fluorescein was used throughout the study. A single buffer, $30 \mathrm{mM}$ ammonium acetate $\mathrm{pH}$ 7.5, was utilized to prepare all solutions and used as the sample propagation buffer; accordingly, we simply refer to it as the buffer.

Protein-ligand EMs were prepared by mixing appropriate volumes of working solutions of $\mathrm{P}$ and $\mathrm{L}$ and incubating for $1 \mathrm{~h}$ before the start of ACTIS runs (longer incubation was shown not to change the determined value of $K_{\mathrm{d}}$ suggesting that $1 \mathrm{~h}$ was sufficient to approach equilibrium in the binding reaction (eq 1)). All EMs had identical total ligand concentration $[\mathrm{L}]_{0}=100 \mathrm{nM}$, unless otherwise stated, while the total protein concentrations in the EMs, $[\mathrm{P}]_{0}$, varied within four orders of magnitude: from $0.1 \mu \mathrm{M}$ to $1 \mathrm{mM}$. All sample handling and measurements were carried out at a room temperature of $20 \pm 2{ }^{\circ} \mathrm{C}$. Identical samples were utilized when experiments were conducted with two ACTIS setups in parallel.

Fluidic Setup Components. The two ACTIS instruments used in this study had similar fluidic systems shown schematically in Figure 2. The fluidic systems included: a pump (called here the main pump, model "AF1", Elveflow, Paris, France), a syringe pump (interchangeably called the sample pump, model "Pump 11 Elite", Harvard Instruments, Holliston, US), a high-pressure 6port 2-position valve (Vici Valco, Houston, TX, USA), a flow rate sensor (model "Coriolis Flow Sensor", Elveflow, Paris, France), and a fluorescence detector (see the details in the next section). Fused-silica capillaries (Molex, Phoenix, AZ, USA) were used for both the separation capillary $(200 \mu \mathrm{m} \mathrm{ID,} 80 \mathrm{~cm}$ length $)$ and the injection loop (100 $\mu \mathrm{m}$ ID, $13 \mathrm{~cm}$ length; $1 \mu \mathrm{L}$ volume). Fittings and connectors were from SciPro Technologies (Sanborn, US). The main pump could be operated in both pressure-control mode and flow-rate-control mode. We found that this pump produced a more stable flow when operated in the pressure-control mode while the flow rate was the parameter to be controlled. Therefore, the pump pressure $P$ was changed to obtain a desired flow rate $Q$ measured by the flow-rate sensor (see the numerical details below). The syringe pump controlled the flow rate of the sample inflow into the valve at a constant level of $Q_{\mathrm{s}}$ (see the numerical details below). No special effort was taken to make the fluidic systems of the two ACTIS instruments identical. Accordingly, there were likely some minor variations allowing us to test if such variations could affect $K_{\mathrm{d}}$ determination significantly. Photographs of both instruments are shown in Figure S1.

Fluorescence Detection. Laser-induced-fluorescence detectors from commercial $\mathrm{CE}$ instruments (model "PACEMDQ", SCIEX, Concord, Ontario, Canada) were used for the two ACTIS setups. Both utilized $488 \mathrm{~nm}$ solid-state lasers (Model W488-08PM, Pavilion Integration Corporation, San Jose, CA, USA). Fluorescence signals were detected through transparent windows (detection windows) in the uncoated (non-transparent) capillaries; the detection windows were located $60 \mathrm{~cm}$ from the capillary inlet. These detectors use ball lenses to collect fluorescence light. The lens is placed in close proximity to the capillary. Focusing is very sensitive to the lens position, and it may differ from detector to detector. No effort was taken to make focusing identical for the two detectors.

ACTIS Experiment. The same experimental procedure was used for both ACTIS instruments. The fluidic setup was prefilled with the buffer before the first run in a run series with one concentration of the protein. A standard ACTIS experiment included four steps facilitated by two positions (I and II) of the valve and two pumps (Figure 2). In Step 1, the valve was in position I, and the injection loop was filled continuously with the sample using a syringe pump at a flow rate of $Q_{\mathrm{s}}=20 \mu \mathrm{L} / \mathrm{min}$. The main pump was running at a pressure $P_{\text {inj }}$ identical to that of

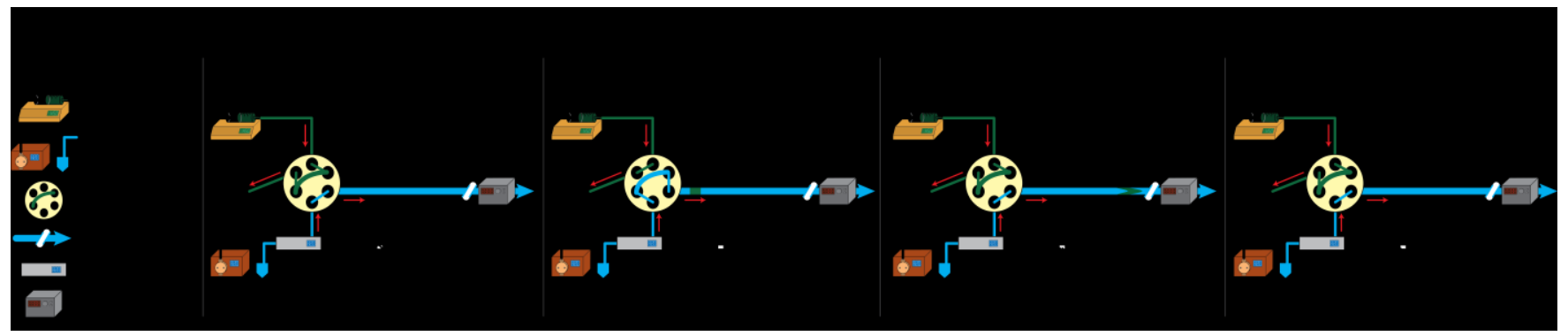

Figure 2. Components of the ACTIS setup and four steps of a single run in an ACTIS experiment, which produces a single separagram. The two valve positions are indicated by Roman numbers I and II. Letters $P$ and $Q$ show pressures and flow rates, respectively. The red arrows show the directions of the flows. See the text for more details. 
the next step. In Step 2, the valve was switched to position II, and the sample plug was slowly transferred from the injection loop to the separation capillary by the main pump for $24 \mathrm{~s}$ at a pressure $P_{\text {inj }}$ set at a level that produced a flow rate of $Q_{\text {inj }}=5 \mu \mathrm{L} / \mathrm{min}$. When the transfer ended, the sample plug was one sample-plug distance from the separation-capillary inlet to ensure a complete sample transfer to the separation capillary. In Step 3, the valve was switched back to position I, and the sample plug was propagated inside the capillary for $48 \mathrm{~s}$ by the main pump at a pressure of $P_{\mathrm{TIS}}$ that produced a flow rate of $Q_{\mathrm{TIS}} \approx 57 \mu \mathrm{L} / \mathrm{min}$. $Q_{\text {TIS }}$ was chosen to allow efficient TIS of L from the PL (see Note S1 for calculations). In Step 4, the valve remained in position I, and the separation capillary was rinsed with the buffer, which was pumped by the pressure pump at a flow rate of $Q_{\text {rinse }} \approx 250 \mu \mathrm{L} / \mathrm{min}$ for $1 \mathrm{~min}$ allowing for 10 volumes of the separation capillary to pass through the fluidic system. The fourstep experimental run took approximately $2.3 \mathrm{~min}$.

Before injecting a sample with a different protein concentration, the fluidic system was thoroughly rinsed with the buffer in a final rinsing step (see Note S2 for details). For this "between-sample" rinsing step, a syringe containing the buffer was mounted on a syringe pump set to run at a flow rate of $80 \mu \mathrm{L} / \mathrm{min}$, and the main pump kept injecting the buffer at a pressure corresponding to a flow rate of $80 \mu \mathrm{L} / \mathrm{min}$; the valve was in position II. The buffer was pumped through two ports of the valve by repeatedly switching the valve between positions I and II. For each switch the valve spent $1 \mathrm{~s}$ in position I (the time for the injection loop to be filled once and) and $2 \mathrm{~s}$ in position II (allowing the injection of two volumes of the injection loop into the separation capillary). This switching mimicked the sampleinjection and TIS steps of the ACTIS experiment allowing the buffer to attain all the parts inside the fluidic system where the sample might have been retained or adsorbed. During this rinsing, nine volumes of the separation capillary passed through the fluidic system. This rinsing step took approximately $4 \mathrm{~min}$.

Three to five ACTIS runs were performed for each concentration of the protein to establish the mean value and standard deviation. An ACTIS experiment with 10 protein concentrations along with all the rinsing steps took approximately $3.5 \mathrm{~h}$.

Determination of $K_{\mathrm{d}}$. The values of $K_{\mathrm{d}}$ were determined by (i) building a classic binding isotherm "fraction of free ligand $R v \mathrm{~s}$ protein concentration $[\mathrm{P}]_{0}$ " and $(i i)$ performing a non-linear fitting of the theoretical dependence of $R$ on $[\mathrm{P}]_{0}$, in which $K_{\mathrm{d}}$ is a fitting parameter, into the experimental isotherm. ${ }^{2}$ To obtain the $R$ values, we performed TIS on each of the 10 EMs (each with a different total concentration of the protein $[\mathrm{P}]_{0}$ ) by running each EM in the ACTIS instrument as described in the previous section. At least three replicated runs were made for each EM, and a signal value for each run was obtained by averaging points within a time window around the diffusive-peak-maximum position. This position corresponds approximately to the characteristic time of transverse diffusion of the protein-unbound ligand. ${ }^{2}$ We took a total time window width corresponding to $8 \%$ of the diffusivepeak-maximum position, i.e., $\pm 4 \%$ around this position. The time window used in the determination of signal values was selected from the first $\mathrm{EM}$ at $[\mathrm{P}]_{0}=0.1 \mu \mathrm{M}$ and applied subsequently to all other EMs at other $[\mathrm{P}]_{0}$ values. The signal values obtained from the replicated runs were used to find an average signal value, from which the values of $R$ and corresponding standard deviations (obtained through simple error propagation) were calculated using equations described in a previous ACTIS publication. ${ }^{2}$ The $K_{\mathrm{d}}$ value and its standard deviation were then obtained by fitting the experimental dependence of $R$ vs $[\mathrm{P}]_{0}$ with their theoretical relationship by performing a non-linear fitting using the Levenberg-Marquardt algorithm in the OriginPro software. The standard deviation of $K_{\mathrm{d}}$ values indicates the goodness of the nonlinear fit.

\section{RESULTS AND DISCUSSION}

An ACTIS instrument has a minimum fluidic setup with the sample pump, the main pump, the injection loop, the separation capillary, the multiport valve, and the flow-rate sensor (Figure 2). The deterministic nature of ACTIS allowed us to assess its accuracy earlier by using a virtual ACTIS instrument built in COMSOL. ${ }^{7}$ Our in-silico study revealed that ACTIS accuracy was not influenced significantly by large variations in the parameters of the fluidic setup: the radius of the separation capillary, the radius and length of the injection loop, and the ramp time of the main pump. ${ }^{7}$ This invariance of accuracy is important because it gives flexibility in instrument design, and guarantees that the accuracy will not be affected by deviations in the performance parameters of the key instrument components from their nominal values provided by the manufacturer. Specifically, this robustness of accuracy suggests that a very elaborate fluidic system used by us in the proof-of-concept work to achieve a near-cylindrical starting sample plug is not necessary. ${ }^{2,7}$ Therefore, we assembled a physical mimic of the simple fluidic setup evaluated in the insilico study (see Materials and Methods for details).

ACTIS is a titration-like method with no internal standard. Therefore, the high repeatability of separagrams obtained from injecting identical samples is imperative for high accuracy in $K_{\mathrm{d}}$ determination. Two conditions must be satisfied to achieve run-torun repeatability: ( $i$ ) no sample carryover and (ii) long-term operation stability of all components of the instrument, specifically the main pump and the detector. Sample carryover and instrument instabilities can cause gradual changes in separagrams which will inevitably lead to systematic errors in $K_{\mathrm{d}}$ values. Observing repeatability of separagrams and signal stability would indicate that both conditions are satisfied, i.e., there is neither sample carryover nor long-term operation instability of instrument components.

The stability of the ACTIS instrument was proven by conducting 60 consecutive runs of a fluorescein solution for approximately $2 \mathrm{~h}$. The resulting separagrams and dependencies of the peak parameters (height and position) on run number for the first ACTIS instrument are shown in Figure 3A, left and right panels, respectively. The dependencies show approximately $5 \%$ decrease in peak height towards a stable value during the first 10 runs and only a random variation $(2 \% \mathrm{RSD})$ in peak position. The initial change in peak height is likely associated with laser's and/or photomultiplier's slowly reaching their stable operation condition, e.g., the steady-state temperature. The absence of any noticeable drifts in peak position suggests very stable operation of the main pump. To increase the stringency of this test, we then ran a mixture containing $0.5 \mathrm{mM}$ concentration of BSA along with fluorescein. BSA is a highly-adsorbing protein and the $0.5 \mathrm{mM}$ concentration used is among the highest concentrations ever used in in-vitro experiments. This mixture was a perfect sample to challenge the ACTIS instrument and check if there was any sample carryover caused by protein adsorption. We conducted 60 consecutive runs of this mixture over a period of approximately $2 \mathrm{~h}$ (on a different day) and found highly repetitive separagrams (Figure 3B, left). Heights and positions of the diffusive peak were measured and presented as functions of run number (Figure 3B, right). The dependencies were similar to those of pure fluorescein (Figure 3A, right), leading to similar conclusions about the performance of the detector and the main pump: minor relaveling in the detector response and no notisable instability in the main pump pressure.

The observed stability of separagrams for samples without and with the protein indicates that there was no sample carryover and that the pump and detector could support stable instrument operation. Specifically, these results suggest that the rinsing procedure used by us will be adequate for most other proteins as BSA is a highly-adsorbing protein. ${ }^{10}$ In the unlikely event that 

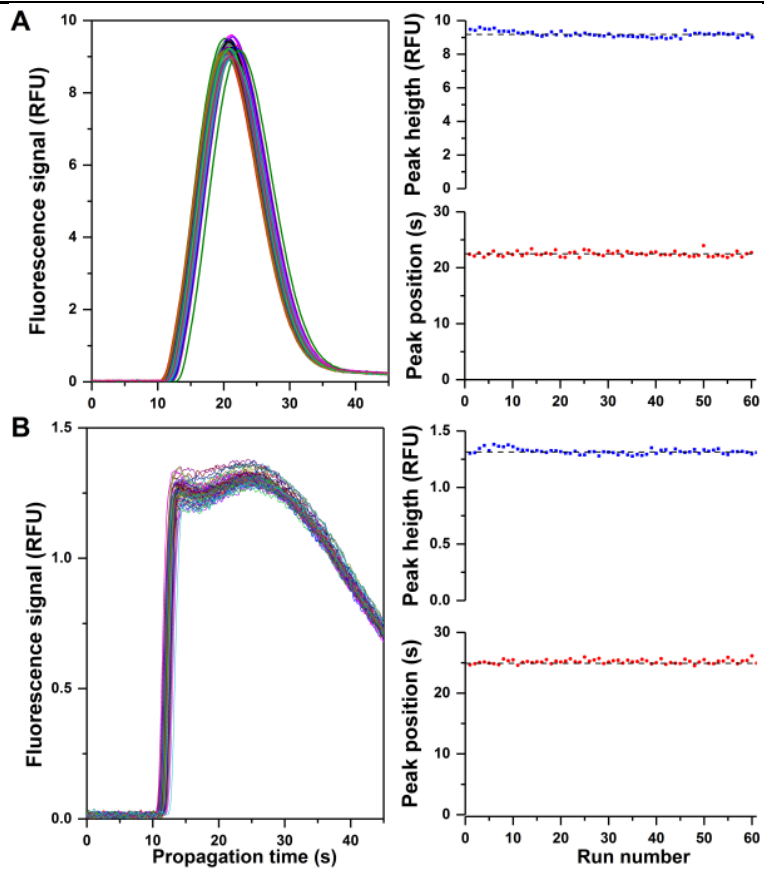

Figure 3. Repeatability of separagrams in 60 consecutive runs of $100 \mathrm{nM}$ fluorescein (A) and a mixture of $100 \mathrm{nM}$ fluorescein with $0.5 \mathrm{mM}$ BSA (B). The left panels show separagrams while the right panels show the dependencies of the height and position of the diffusive peak on the run number.

protein adsorption for another protein is observed, the inner surface of the injection loop and the separation capillary can be coated to suppress protein adsorption. ${ }^{11}$

Finally, we conducted a comprehensive test of ACTIS ruggedness. We assembled the second ACTIS instrument and ran ACTIS experiments on two instruments by two operators in parallel using identical samples for every experimental set. The instruments were built of similar components, but no specific effort was undertaken to make the fluidic systems and detectors identical. As a result, the instruments likely differed in their ratios of the capillary length to flow velocity; however, ACTIS is robust to variations in this parameter. ${ }^{7}$ The instruments also likely
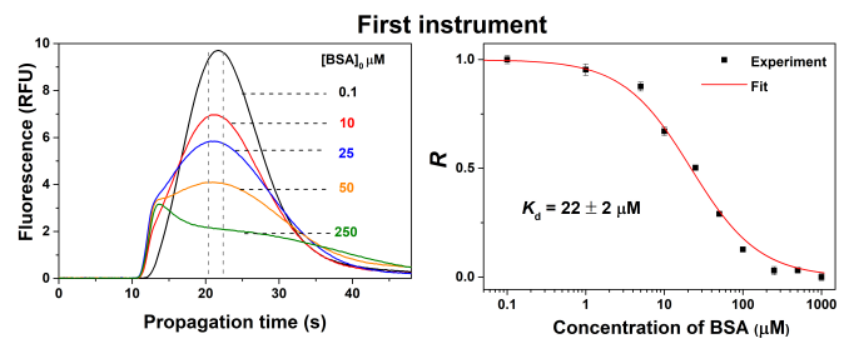

Second instrument

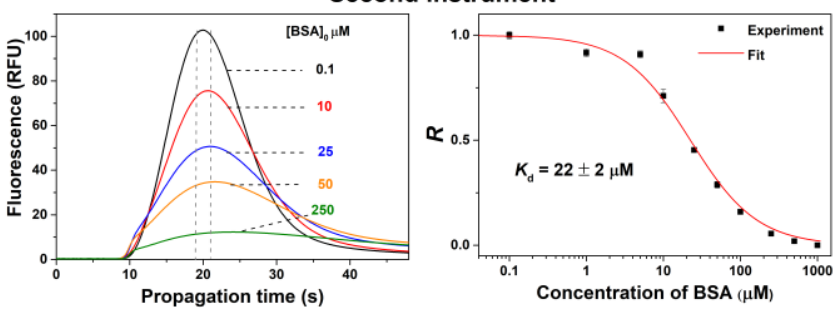

Figure 4. Robustness of $K_{\mathrm{d}}$ determination by ACTIS demonstrated with two ACTIS instruments used to run identical samples containing $100 \mathrm{nM}$ fluorescein and varying concentrations of BSA. The left panels show representative separagrams (one of five for each BSA concentration), and the right panels show the corresponding binding isotherms. $R$ is a fraction of unbound fluorescein.

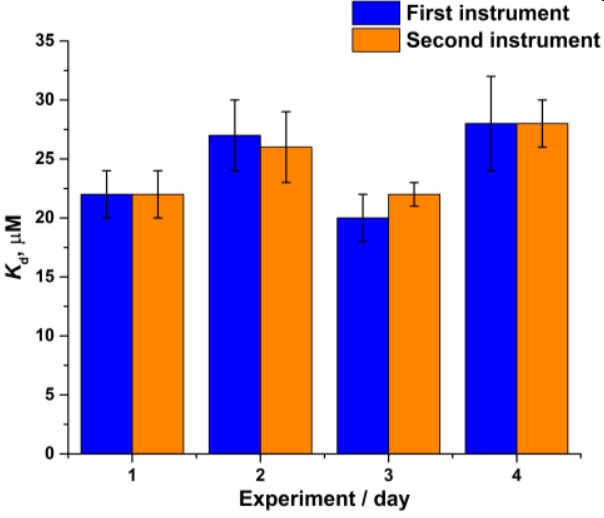

Figure 5. Ruggedness of $K_{\mathrm{d}}$ determination with two ACTIS instruments with new samples prepared for each experiment; identical samples were used for each of the two instruments in each experiment.

differed in focusing performance of the commercial detectors' optics due to the specifics of ball lenses. ${ }^{12}$ These differences cumulatively were the reason for significant differences in the relative heights and positions of the non-diffusive peak (the lefthand side peak) between the two instruments (Figure 4, left panels). The same differences were observed between the results obtained with these instruments in all the following experiments (Figures S2-S7). Importantly, these differences in the separagram shapes did not lead to deviations in the $K_{\mathrm{d}}$ values between the two instruments. All experiments performed in parallel with the two instruments returned $K_{\mathrm{d}}$ values equal within limits of precision provided that identical samples were used (Figure 5). New solutions were prepared every day and day-to-day variations were more significant - the largest $K_{\mathrm{d}}$ value was $28 \mu \mathrm{M}$ and the smallest $20 \mu \mathrm{M}$. Our results clearly show the robustness and ruggedness of ACTIS experiments suggesting that this simple ACTIS instrumentation can support accurate $K_{\mathrm{d}}$ determination.

\section{CONCLUDING REMARKS}

We present a simple ACTIS instrument that supports stable operation, repeatability, and reproducibility. The proven stability of ACTIS instrumentation translates into high accuracy of $K_{\mathrm{d}}$ determination. Our results with two ACTIS instruments suggest that ACTIS instrumentation is responsible for a small inaccuracy (approximately 10\%) in $K_{\mathrm{d}}$ determination. It should be noted that the accuracy of the determined $K_{\mathrm{d}}$ values depends on the accuracy of protein concentrations used. Errors in protein concentrations are the most probable source of inaccuracy in ACTIS experiments manifested in $K_{\mathrm{d}}$ differences between experiments performed with new samples. The influence of inaccurate protein concentration will be more significant for unstable proteins. To address this problem, one can assess the applicability to ACTIS of a doubletitration procedure, in which both the protein concentration in the stock solution and th e $K_{\mathrm{d}}$ value are determined. ${ }^{13}$ To conclude, the simple fluidic setup of the ACTIS instrument reported in this work can be used to accurately and reliably determine $K_{\mathrm{d}}$ values, and we foresee ACTIS being used as a new reference method for $K_{\mathrm{d}}$ measurement.

\section{ASSOCIATED CONTENT}

\section{Supporting Information}

The Supporting Information is available free of charge at ChemRxiv.

Flow Rate for Transient Incomplete Separation (Note S1); Between-sample rinsing step (Note S2); Photographs of the two ACTIS instruments (Figure S1); Determination of $K_{\mathrm{d}}$ value of the BSA-Fluorescein Pair on Day 2 (Figure S2); Determination of $K_{\mathrm{d}}$ value of the BSA-Fluorescein Pair on 
Day 3 (Figure S3); Determination of $K_{\mathrm{d}}$ value of the BSAFluorescein Pair on Day 4 (Figure S4); Determination of $K_{\mathrm{d}}$ value of the BSA-Fluorescein Pair on Day 5 (Figure S5); Repeatability of the ACTIS Separagrams for the BSAFluorescein Complex, Day 1, Instrument 1 (Figure S6); Repeatability of the ACTIS Separagrams for the BSAFluorescein Complex, Day 1, Instrument 2 (Figure S7).

\section{AUTHOR INFORMATION Corresponding Author \\ *E-mail: skrylov@yorku.ca. \\ ORCID}

$\begin{array}{ll}\text { Sergey N. Krylov: } & 0000-0003-3270-2130 \\ \text { J.C. Yves LeBlanc: } & 0000-0002-3801-3590 \\ \text { Jean-Luc Rukundo: } & 0000-0003-3626-2515 \\ \text { Sven Kochmann: } & 0000-0001-7423-4609 \\ \text { Nikita A. Ivanov: } & 0000-0002-0842-6626 \\ \text { Tong Y. Wang: } & 0000-0001-9462-7194 \\ \text { Boris I. Gorin: } & 0000-0003-1500-5392\end{array}$

\section{Author Contributions}

The manuscript was written through contributions of all authors, and all authors have given approval to the final version of the manuscript before the submission.

\section{Notes}

The authors declare no competing financial interest.

\section{ACKNOWLEDGMENTS}

This work was supported by the Natural Sciences and Engineering Research Council of Canada (Grant SPGP 521331-2018).

\section{REFERENCES}

(1) Chandran, S.; Singh, RS. Comparison of various international guidelines for analytical method validation. Pharmazie. 2007, 62:4-14.

(2) Sisavath, N.; Rukundo, J.-L.; Le Blanc, J. C. Y.; Galievsky, V. A.; Bao, J.; Kochmann, S.; Stasheuski, A. S.; Krylov, S. N. Transient Incomplete Separation Facilitates Finding Accurate Equilibrium Dissociation Constant of ProteinSmall Molecule Complex. Angew. Chem. Int. Ed. 2019, 58, 6635-6639.

(3) Bottari, F.; Daems, E.; de Vries, A. M.; Van Wielendaele, P.; Trashin, S.; Blust, R.; Sobott, F.; Madder, A.; Martins, J. C.; \& De Wael, K. Do Aptamers Always Bind? The Need for a Multifaceted Analytical Approach When Demonstrating
Binding Affinity between Aptamer and Low Molecular Weight Compounds. JACS, 2020, 142, 19622-19630.

(4) Fielding, L. NMR methods for the determination of protein-ligand dissociation constants. Prog. Nucl. Mag. Res. Sp. 2007, 51, 219-242.

(5) Watzig, H.; Oltmann-Norden, I.; Steinicke, F.; Alhazmi, H. A.; Nachbar, M.; El-Hady, D. A.; Albishri, H. M.; Baumann, K.; Exner, T.; Bockler, F. M.; El Deeb, S. Data Quality in Drug Discovery: the Role of Analytical Performance in Ligand Binding Assays. J. Comput. Aided Mol. Des. 2015, 29, 847-865.

(6) Hulme, E. C.; Trevethick, M. A. Ligand binding assays at equilibrium: validation and interpretation. Br. J. Pharmacol. 2010, 161, 1219-37.

(7) Rukundo, J-L.; Le Blanc, J. C. Y.; Kochmann, S.; Krylov, S. N. Assessing Accuracy of an Analytical Method in silico: Application to "Accurate Constant via Transient Incomplete Separation" (ACTIS). Anal. Chem. 2020, 92, 11973-11980.

(8) Harada, M.; Kido, T.; Masudo, T.; Okada, T. Solute Distribution Coupled with Laminar Flow in Wide-Bore Capillaries: What Can Be Separated without Chemical or Physical Fields? Anal. Sci. 2005, 21, 491-496.

(9) Okada, T.; Harada, M.; Kido, T. Resolution of Small Molecules by Passage through an Open Capillary. Anal. Chem. 2005, 77, 6041-6046.

(10) Scarangella, A., Soumbo, M., Villeneuve-Faure, C., Mlayah, A., Bonafos, C., Monje, M. C., Roques, C., \& Makasheva, K. Adsorption properties of BSA and DsRed proteins deposited on thin SiO2layers: optically non-absorbing versus absorbing proteins. Nanotechnology. 2018, 29(11), 115101.

(11) Yu, B.; Liu, P.; Cong, H.; Tang, J.; Zhang, L. Novel covalently coated diazoresin/polyvinyl alcohol capillary column for the analysis of proteins by capillary electrophoresis. Electrophoresis. 2012, 33, 3066-3072.

(12) Nouadje, G.; Nertz, M.; Verdeguer, P.; Couderc, F. Balllens laser-induced fluorescence detector as an easy-to-use highly sensitive detector for capillary electrophoresis - application to the identification of biogenic-amines in dairy-products. $J$. Chromatogr. A. 1995, 777, 335e 343 .

(13) Ye, N.; Busch, D. H. Modeling the heteroleptic equilibria of organized molecular systems by using a double-titration technique: a novel determination of the fundamental equilibrium constants in a ternary system involving a macrocyclic cobalt (II) complex, dioxygen, and competing axial ligands. Inorg. Chem. 1991, 30, 1815-1819. 